\title{
Study of the Electron-Positron Annihilation Coincidence Peak Two-Dimensional Profile
}

\author{
E. do Nascimento, O. Helene, V. R. Vanin, \\ Instituto de Física, Universidade de São Paulo, C.P. 66318, 05389-970, São Paulo, SP, Brazil \\ and C. Takiya \\ Departamento de Ciências Exatas, Universidade Estadual do Sudoeste da Bahia, Vitória da Conquista, BA, Brazil
}

Received on 20 October, 2003

\begin{abstract}
Positron annihilation radiation profile in aluminum was observed with a pair of Ge detectors in coincidence.
${ }^{22} \mathrm{Na}$ was used as a source of positron and the two-dimensional gamma energy spectrum was fitted using a model function. Annihilation components of positron at rest with conduction band, $1 \mathrm{~s}, 2 \mathrm{~s}$, and $2 \mathrm{p}$ electrons were observed. The in-flight positron annihilation was also observed. The model function also took into account the detector response function, relative efficiency corrections and the gamma backscattering. Coincidences involving a combination of Compton effect, pileup, ballistic deficit, and pulse shaping problems were treated as well.
\end{abstract}

\section{Introduction}

This study aimed to understand the shape of the electronpositron annihilation peak measured in coincidence by two photon detectors (Fig. 1).

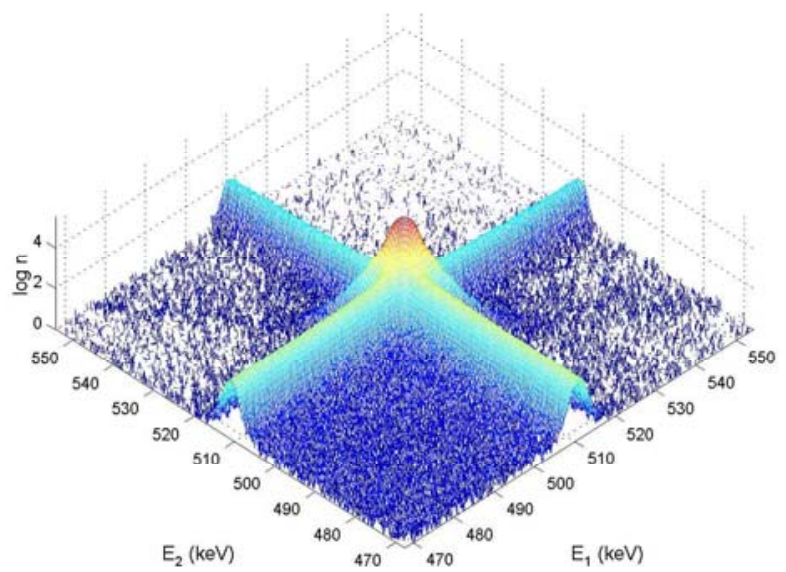

Figure 1. Distribution of coincident events as a function of the measured energies $E_{1}$ and $E_{2}$, showing the Doppler broadening.

The proper fit of the $511 \mathrm{keV}-511 \mathrm{keV}$ peak requires many analytical functions, giving information about the electron momentum distribution in the analyzed material. This technique is known as Coincidence Doppler Broadening $(\mathrm{CDB})$ of the electron-positron annihilation radiation [1] and is used in studies of the electronic and atomic strutures of defects in solids $[2,3,4]$.

\section{Experimental Setup}

The profile of the annihilation peak of positrons from $\mathrm{a}^{22} \mathrm{Na}$ source in metallic Al was measured with the Linear Accelerator Laboratory residual radioactivity multi-detector array (MULTI) [5]. The two annihilation gamma-rays were measured with a pair of Ge detectors in coincidence, placed in diametrically opposed positions, separated by $15 \mathrm{~cm}$, and with a $3.7 \times 10^{5} \mathrm{~Bq}(10 \mu \mathrm{Ci}){ }^{22} \mathrm{Na}$ source. This source was placed between two $2 \mathrm{~mm}$ thick aluminum sheets $(99.999 \%$ pure). An ${ }^{192}$ Ir source was simultaneously measured to provide references for detector calibration and follow any energy calibration drift during the experiment. The measurement run lasted for $200 \mathrm{~h}$, when $1.5 \times 10^{7}$ events in the peak region were accumulated.

\section{Model Function}

Usually the results of Doppler broadening measurement are analyzed comparing the calculated annihilation probability density with the experimental data. In this work we opted for another procedure. The convolution of the detector response function with empirical functions to represent the gammarays emitted after positron annihilation with $1 \mathrm{~s}, 2 \mathrm{~s}, 2 \mathrm{p}$ and conduction electrons were calculated. All these functions were parametrized. This procedure avoids the dificult problem of deconvolution of the Doppler broadening spectrum [6] .

The function model was determined from a qualitative analysis of the experimental data and published theoretical results $[7,8]$. Positron annihilation with band electrons was fitted by three arcs of parabola and one gaussian along the 
line $E_{1}+E_{2}=1022 \mathrm{keV}$ :

$f_{b}=\sum_{i=1}^{3} C_{i}\left(E_{1}-E_{2}-\alpha_{i}\right)\left(E_{1}-E_{2}+\alpha_{i}\right)+\frac{A_{b} e^{\frac{-\left(E_{1}-E_{2}\right)^{2}}{2 \sigma_{b}^{2}}}}{\sqrt{2 \pi} \sigma_{b}}$

where $E_{1}$ and $E_{2}$ are energies in detectors 1 and 2 respectively, and $\alpha_{i}$ are the cutoff parameters $\left(C_{i}=0\right.$ when $\left|E_{1}-E_{2}\right|>\alpha_{i}$ ). Positron annihilation with 1 s electrons was fitted by one gaussian along the line $E_{1}+E_{2}+B_{1 s}=1022$ $\mathrm{keV}$ :

$$
f_{1 s}=\frac{A_{1 s} e^{\frac{-\left(E_{1}-E_{2}\right)^{2}}{2 \sigma_{1 s}^{2}}}}{\sqrt{2 \pi} \sigma_{1 s}}
$$

where $B_{1 s}$ is the binding energy of the 1 s electrons. Positron annihilattion with 2 s electrons was fitted by two gaussians along the line $E_{1}+E_{2}+B_{2 s}=1022 \mathrm{keV}$ :

$$
f_{2 s}=\frac{A_{2 s} e^{\frac{-\left(E_{1}-E_{2}\right)^{2}}{2 \sigma_{2 s}^{2}}}}{\sqrt{2 \pi} \sigma_{2 s}}+\frac{A_{2 s}^{\prime} e^{\frac{-\left(E_{1}-E_{2}\right)^{2}}{2 \sigma_{2 s}^{\prime 2}}}}{\sqrt{2 \pi} \sigma_{2 s}^{\prime}}
$$

where $B_{2 s}$ is the binding energy of the 2 s electrons. Positron annihilation with $2 \mathrm{p}$ electrons was fitted by one gaussian along the line $E_{1}+E_{2}+B_{2 p}=1022 \mathrm{keV}$ :

$$
f_{2 p}=\frac{A_{2 p} e^{\frac{-\left(E_{1}-E_{2}\right)^{2}}{2 \sigma_{2 p}^{2}}}}{\sqrt{2 \pi} \sigma_{2 p}}
$$

where $B_{2 p}$ is the $2 \mathrm{p}$ electron binding energy. Finally, inflight positron annihilation was fitted by:

$$
f_{f}=\frac{A_{f} e^{-\lambda d} e^{\frac{-\left(E_{1}-E_{2}\right)^{2}}{2 \sigma_{f}^{2}}}}{\sqrt{2 \pi} \sigma_{f}}
$$

where

$$
d=\frac{m_{0} c^{2}}{\sqrt{2}}-\sqrt{\left(E_{1}-\frac{3 m_{0} c^{2}}{2}\right)^{2}+\left(E_{2}-\frac{3 m_{0} c^{2}}{2}\right)^{2}}
$$

(Fig. 2). The $A^{\prime} s$ and $\sigma$ 's are the areas and widths of the gaussians respectively. Detection effects due to ballistic deficit, pile-up and Compton scattering (Fig. 3) were considered in the fit. Coincidences involving a combination of Compton effect, pileup, ballistic deficit, and pulse shaping problems (Fig. 4), backscatering (Fig. 5) and efficiency corrections of the detectors in the fitting region, were taken into account. The model functions were fitted to the experimental data and the result is shown in Fig. 6.

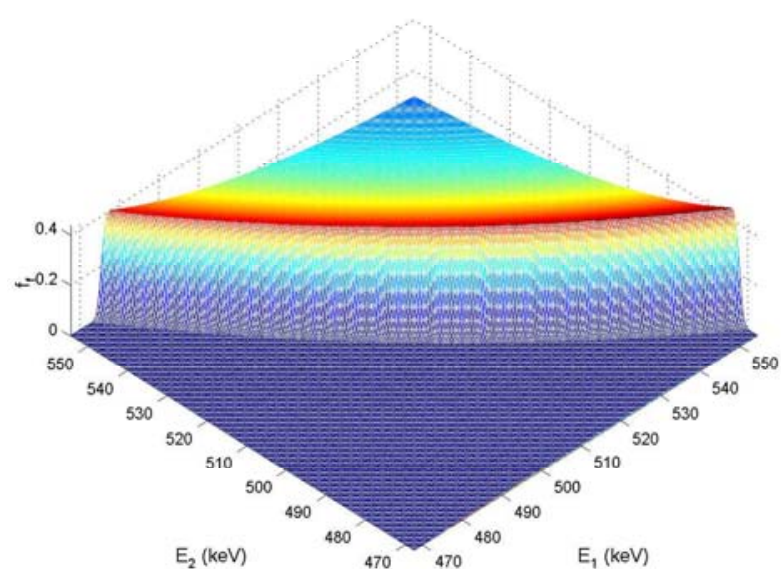

Figure 2. Two-dimensional representations of in-flight positron annihilation radiation.
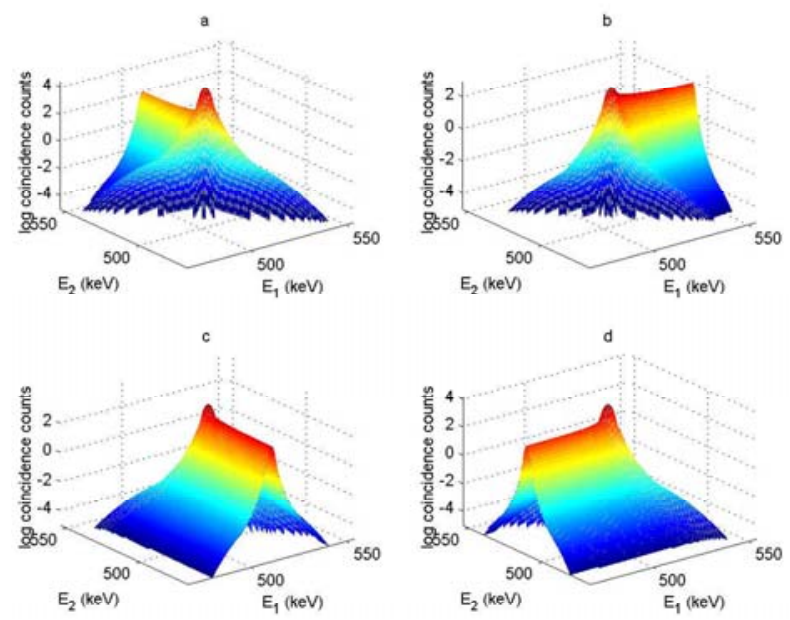

Figure 3. Two-dimensional representations of the fitted exponential tails of the electron-positron annihilation peak. The internal exponentials tails for detectors 1 and 2 are represented in parts (a) and (b) respectively. The external exponential tails for detectors 1 and 2 are in (c) and (d), respectively.

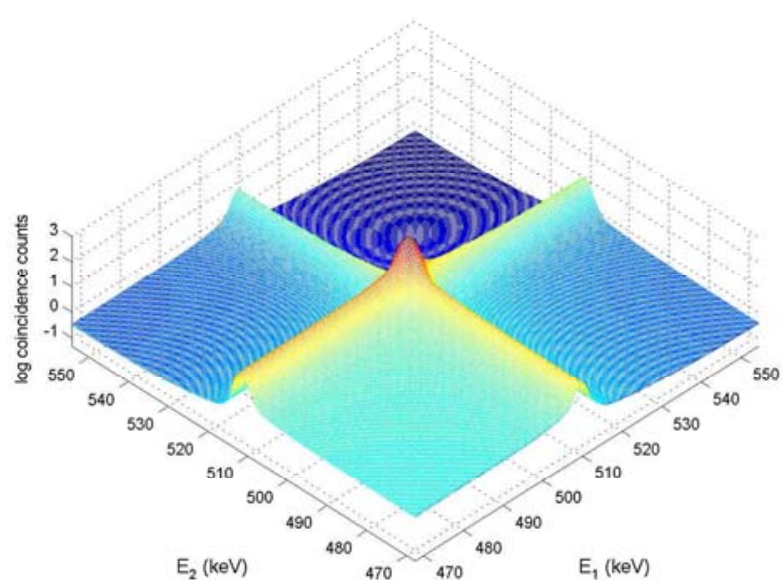

Figure 4. Two-dimensional representations of Compton-Compton and other effects. 


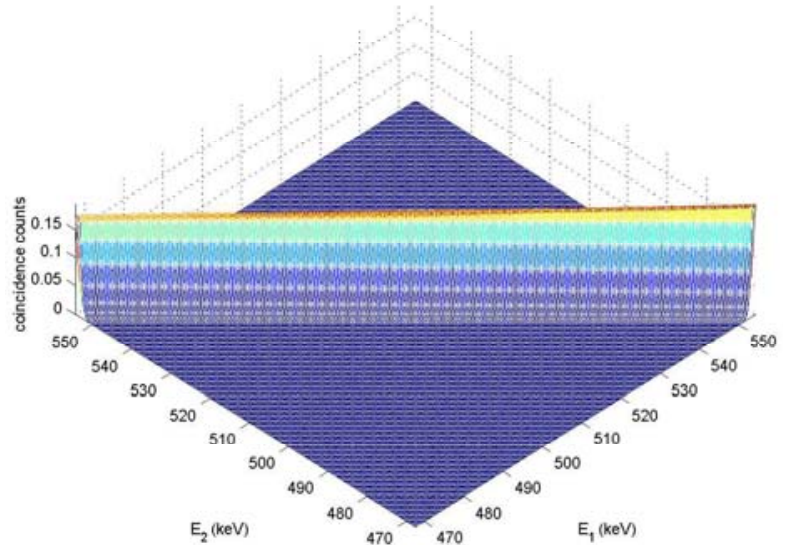

Figure 5. Two-dimensional representations of the backscattering coincidence.

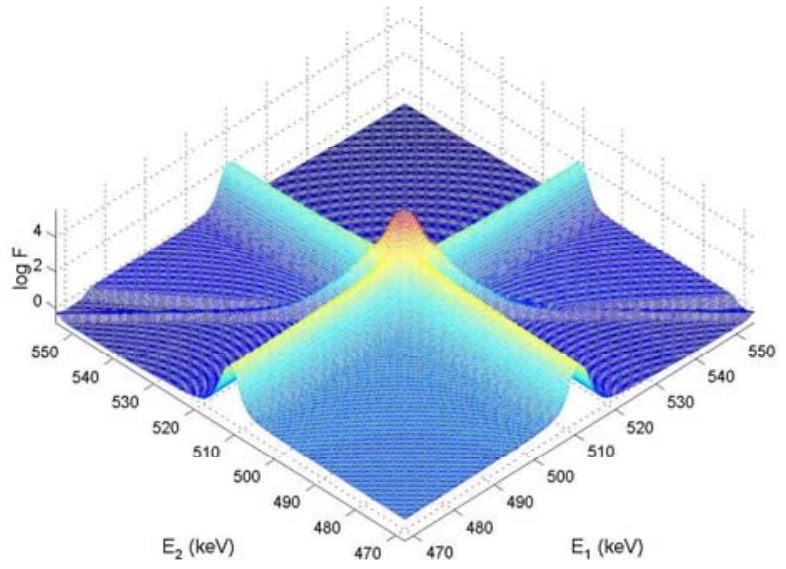

Figure 6. The fitted coincidence spectrum.

The fit was done by the least-squeares method (see, for instance [9]) with the Gauss-Marquardt algorithm due to the non-linearity in the parameters [10]. The chi-squared value was calculated by

$$
\chi^{2}=\sum_{i, j} \frac{\left(n_{i j}-F_{i j}\right)^{2}}{F_{i j}}
$$

where $n_{i j}$ is the number of observed events in channel $(i, j)$ of the coincidence spectrum (Fig. 1), and $F_{i j}$ is the fitted function (Fig. 6).

\section{Conclusion}

The reduced $\chi^{2}$ obtained in this study, 1.1 with about 62448 degrees of freedom, does not show a disagreement between the data and the model, suggesting that a complete statistical analysis of the coincidence Doppler broadening annihilation radiation is possible. Better model functions can be considered in order to improve the $\chi^{2}$ value [1].

\section{Acknowledgments}

We wish to acknowledge the support of Conselho Nacional de Desenvolvimento Científico e Tecnológico CNPq, Fundação de Amparo à Pesquisa do Estado de São Paulo - FAPESP, and the Data Section of the International Atomic Energy Agency.

\section{References}

[1] P. E. Mijnarends, A. C. Kruseman, A. van Veen, H. Schut, and A. Bansil, J. Phys. Condens. Mat. 10(46) 10383 (1998).

[2] M. Hasegawa, Z. Tang, Y. Nagai, T. Nonaka, and K. Nakamura, Appl. Surf. Sci. 194, 76 (2002).

[3] Y. Nagai, Z. Tang, H. Ohkubo, K. Takadate, and M. Hasegawa, Rad. Phys. Chem. 68, 381 (2003).

[4] M. J. Puska and R. M. Nieminen, Rev. Mod. Phys. 66, 841 (1994).

[5] V. R. Vanin, R C Neves, et. al., XIX Reunião de Trabalho sobre Física Nuclear no Brasil (1996)

[6] C. Takiya, O. Helene, E. do Nascimento, V. R. Vanin, Nucl Instr Meth A (2004) in press.

[7] Z. Tang, M. Hasegawa, Y. Nagai, M. Saito, and Y. Kawazoe, Phys. Rev. B 65, 045108 (2002).

[8] V. J. Ghosh, M. Alatalo, P. Asoka-Kumar, B. Nielsen, K. G. Lynn, A. C. Kruseman, and P. E. Mijnarends, Phys. Rev. B 61, 10092 (2000).

[9] V. R. Vanin, G. Kenchian, M. Moralles, O. A. M. Helene, and P. R. Pascholati, Nucl. Instr. Meth. A 391, 338 (1997).

[10] D. W. Marquardt, J. Soc. Appll. Math. 11, 431 (1963). 